\title{
The possible locus of lightness contrast
}

\author{
FREDERICK L: KITTERLE \\ The Unilersity of Toledo, Toledo, Ohio 43606
}

\begin{abstract}
Evidence that the magnitude of simultaneous contrast depends upon retinal orientation of a stimulus configuration is presented in two experiments. More specifically. the magnitude of contrast was found to be greater for stimuli presented horizontally and vertically than for stimuli presented obliquely. These results were interpreted in terms of a cortical analysis of simultaneous contrast.
\end{abstract}

Most physiological explanations of simultaneous contrast have been in terms of lateral interactions within receptive fields of retinal ganglion cells. Recent psychophysical evidence seems to indicate that the analysis of contrast is probably not performed in the retina but, rather, at some higher level within the visual system (Fiorentini \& Maffei, 1973; Hollins, 1971; Gogel \& Mershon, 1969; Mershon, 1972; Wist \& Susen, in press).

At very low luminances (i.e., scotopic levels), marked contrast effects have been obtained (Hollins, 1971; Fiorentini \& Maffei, 1973). In a related experiment, attenuation of the spatial modulation transfer function has been found for very low spatial frequencies at scotopic levels, a fact which has been interpreted to indicate the existence of lateral inhibition (Fiorentini \& Maffei, 1973).

These results would seem to rule out an analysis of contrast at a retinal level, because the results of Barlow, Fitzhugh, and Kuffler (1957) indicate that, at very low luminances, surrounds of receptive fields of retinal ganglion cells disappear and, consequently, lateral inhibitory interactions.

Gogel and Mershon (1969) and Mershon (1972) varied the depth between the test and inducing field and found that contrast varied as a function of the perceived depth interval. This effect was independent of the small lateral displacements on each retina. Wist and Susen (in press) extended these findings to show that the magnitude of simultaneous contrast in the Koffka ring is diminished when the perceived depth interval between the bisecting line and the ring is increased.

These studies indicate that conditions which make the test patch appear at different distances reduces contrast, even though the two regions remain laterally adjacent and unchanged in their luminances. Such changes in simultaneous contrast cannot be accounted for in terms of lateral inhibitory interactions operating at a retinal level. Rather, the depth effect indicates processing at or beyond the level of neural organization responsible for processing binocular depth information. This evidence, and also the fact that dichoptic contrast effects have been obtained which are as strong as those obtained monocularly (Yund, 1970), strongly suggests that contrast effects may be due to cortical processing.

The purpose of the experiments reported in this paper is to provide further evidence that the analysis of contrast may be performed at a cortical level.

\section{EXPERIMENT I}

Neurophysiological evidence has shown that receptive fields of the cat and monkey are tuned to both size and orientation (Hubel \& Wiesel, 1962, 1968). That is, a given cell responds maximally to bars or contours of a particular size and orientation. Departure from these optimal characteristics results in the diminution or elimination of that cell's response. Pettigrew, Nikara. and Bishop (1968) found that neurons of the visual cortex in the area centralis show a preference for horizontal and vertical orientations. That is, the firing of a neuron to a particular stimulus is stronger when it is horizontal or vertical and weaker if the stimulus is presented in an oblique orientation.

Psychophysical studies of acuity and contrast sensitivity on man indicate that the visual system of man is organized rather similarly to that of the cat and monkey. These studies show orientation and size tuning (Campbell \& Kulikowski, 1966; Gilinsky, 1968: Campbell \& Maffei, 1970; Blakemore \& Campbell. $1969)$, as well as preferences for horizontal and vertical stimuli (Leibowitz, 1953; Higgins \& Stultz, 1948).

If cortical receptive fields are involved in determining contrast, then the magnitude of lightness contrast should be stronger in the horizontal and vertical meridians than in the oblique meridian, a supposition tested and verified in Experiment I.

\section{Method}

\section{Apparatus}

The stimuli in this experiment consisted of four cards, half white and half black. The cards differed in whether the contour dividing the white and black regions was horizontal, vertical. right oblique (45 deg). or left oblique (135 deg). The cards were $17.78 \times 17.78 \mathrm{~cm}$ (4 deg $50 \mathrm{~min} \times 4 \mathrm{deg} 50 \mathrm{~min}$ ) and were viewed at a distance of $210.82 \mathrm{~cm}$. A gray bar. $8.89 \mathrm{~cm}(2 \mathrm{deg}$ $26 \mathrm{~min}) \times 5.08 \mathrm{~cm}(1 \mathrm{deg} 24 \mathrm{~min})$, was glued in the middle of each card. with the longer side along the contour dividing the 


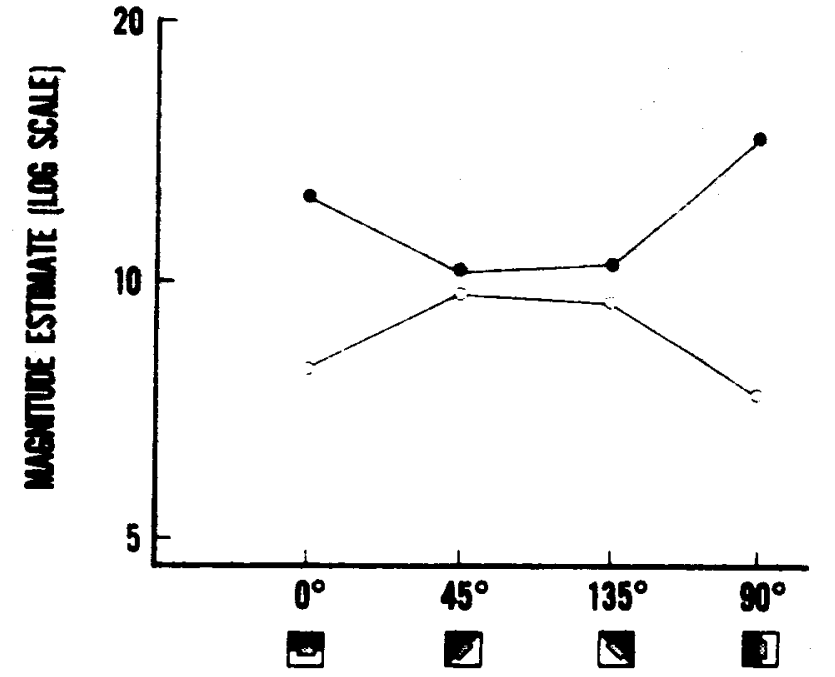

STMMULuS ORERTATION

Fig. 1. Results of Experiment I. Magnitude estimates of the lightness of gray bars on a black (filled circles) and white (open circles) background, as a function of configuration orientation.

black and white regions. Half of it was on the white background and half of it was on the black background. Consequently, the gray bar also appeared in each of the four orientations. A black stripe. $.32 \mathrm{~cm}(6 \mathrm{~min}) \times 8.89 \mathrm{~cm}(2 \mathrm{deg} 26 \mathrm{~min})$, divided the bar into two regions and continued the contour separating the black and white areas through the gray bar.

The cards were illuminated by a Kodak Carousel 8000 projector. Illumination measured with an S.E.I. photometer was $2.8 \log \mathrm{fL}$, and the luminances of the black, white, and gray surfaces were $1.1,2.3$, and $2.0 \log \mathrm{fL}$, respectively. The stimuli were viewed binocularly in an otherwise darkened room.

A fixation point (A) was placed $1.27 \mathrm{~cm}(22 \mathrm{~min})$ above each card. The cards were viewed against a gray background ( $30 \mathrm{deg}$ $20 \mathrm{~min} \times 16 \mathrm{deg} 28 \mathrm{~min}$ ) of $2.0 \mathrm{log} \mathrm{fL}$. Another fixation point (B) was placed in the center of the gray background and $40.64 \mathrm{~cm}(21 \mathrm{deg} 50 \mathrm{~min}$ ) above the middle of the cards.

Fixation Point A placed the test stimulus 2 deg 46 min off the fovea, a retinal region where rather strong contrast effects have been found (Alpern, 1953). Fixation Point B provided a point of reference on the neutral gray adapting field and was sufficiently far from the stimulus cards to rule out any possible influences on the judgments (Alpern, 1953).

\section{Subjects}

Seven $S s$ in this experiment were introductory psychology students, who participated for course credit and were naive with respect to the purpose of the experiment. All Ss had 20/20 vision in each eye and did not wear glasses.

\section{Procedure}

At the beginning of the experimental session, Ss were dark adapted for $10 \mathrm{~min}$ and then light adapted to the gray background. During this time, the procedure was read to the $S$.

The gray on one background served as the modulus, and $\mathrm{Ss}$ judged the lightness of the gray on the opposite background. Thus, with a modulus of 10 , if the gray on the other background was twice as bright as the modulus, it was rated 20 , half as bright. 5. S viewed each card while fixating at $A$ during the 1-sec exposure duration, after which he returned to Fixation Point $B$ until the next trial. All stimulus cards were present throughout the experiment. Eight judgments were made for each card: four with the modulus on the white background and four with the modulus on the black background. Order of stimulus presentation. card location. and modulus location were randomized.

\section{Results}

The results for Experiment I are presented in Fig. 1. Magnitude estimations are plotted as a function of stimulus orientation, with the background (white, open circles; black, filled circles) as the parameter of these curves. Each data point represents the average judgments for the seven Ss. An analysis of variance (Ss by Treatments) was performed on these results.

The fact that the filled points are above the open ones indicates that the backgrounds affected perception of the gray bar. These differences are significant $[F(1,6)=$ $57.54, p<.001]$. Grays against white backgrounds are perceived as darker, grays against black backgrounds are seen as lighter.

The first-order interaction between background and stimulus orientation was also significant $[F(3,18)=$ $15.37, p<.01]$. The apparent lightness differences between the bars changes with stimulus orientation. A greater difference is found for the horizontal and vertical orientations than for the oblique. In other words, the magnitude of contrast changed with stimulus orientation.

It is not clear from these results whether it is orientation in the environment or orientation on the retina which produced the differences in contrast. Experiment II was designed to separate these two effects.

\section{EXPERIMENT II}

If the results obtained in Experiment $I$ are due to orientation in the environment, then viewing the same stimulus configuration with the head oblique or upright should produce no difference in the magnitude of contrast. However, if significant differences are found, they must be due to retinal orientation of the stimulus. For example, an environmentally oblique stimulus viewed with a tilted head would be retinally upright. while with the head upright, the same configuration would be retinally tilted.

\section{Method}

The apparatus for this experiment was essentially the same as that for Experiment $\mathbf{I}$.

\section{Subjects}

Twenty naive Ss participated in this experiment for credit in introductory psychology. All had normal vision and did not wear glasses. They were assigned randomly to one of four experimental groups. The groups differed, depending upon whether $S$ s viewed the stimuli with their heads horizontal. vertical. right oblique $(45 \mathrm{deg})$, or left oblique $(135 \mathrm{deg})$. The 
procedure was essentially the same as that for Experiment I.

\section{Results}

The results of Experiment II are summarized in Fig. 2. In this figure. magnitude estimations are plotted as a function of stimulus orientation for white (open circles) and black (filled circles) backgrounds. The four graphs are for the different head orientations, which are indicated in the upper right-hand corner of each graph. Each data point represents an average of 20 observations ( 5 Ss by 4 replications).

The results of an analysis of variance indicates significant background effects $[F(1,16)=66.80$, $\mathrm{p}<.0001]$. This can be seen in each of the four graphs of Fig. 2, where the filled circles (black backgrounds) are above the open circles (white backgrounds). That is, black backgrounds enhance the lightness of gray bars; white backgrounds depress the lightness of the same bar.

The main effects of stimulus orientation and head orientation are not statistically significant $[F(3,16)=$ .54 . $p>.05$, and $F(3,16)=.86, p>.05$, respectively $]$. This is due to the fact that the lightness judgments on black vs white backgrounds are mirror images of each other as a function of both variables. Thus, they tend to average out close to 10 .

The significant second-order interaction between head orientation. stimulus orientation, and background lightness $[F(9.48)=15.02, p<.01]$ indicates that lightness contrast depends not only on stimulus orientation. but also on head orientation. As seen in Fig. 2, with the head horizontal or vertical, lightness contrast appears strongest for stimuli oriented horizontally and vertically and weaker for oblique orientations, similar to the results in Experiment $I$. However. with the head in an oblique orientation, there is a reversal; contrast is stronger for stimuli oriented obliquely and weaker for stimuli in the horizontal and vertical orientations.

This conclusion was verified by a finer analysis of the second-order interaction. Data for the horizontal and vertical head orientations were pooled, as was the data for the oblique right and oblique left head orientations. For each of the two sets of pooled data, data for horizontal and vertical stimulus orientations were pooled. as was the data for the oblique stimulus orientations.

Against a white background, with the head vertically or horizontally positioned, the magnitude of contrast for the gray bar was stronger in the horizontal or vertical orientations (7.18) compared with the magnitude of contrast for the oblique orientations (9.42). The difference between these two stimulus orientations was statistically significant $[F(1,48)=8.33, p<.025]$. With black background and similar head orientation, the mean judgments for horizontal-vertical vs oblique was 13.72 and 11.07. respectively, a statistically significant difference $[F(1.48)=12.36, p<.025]$.

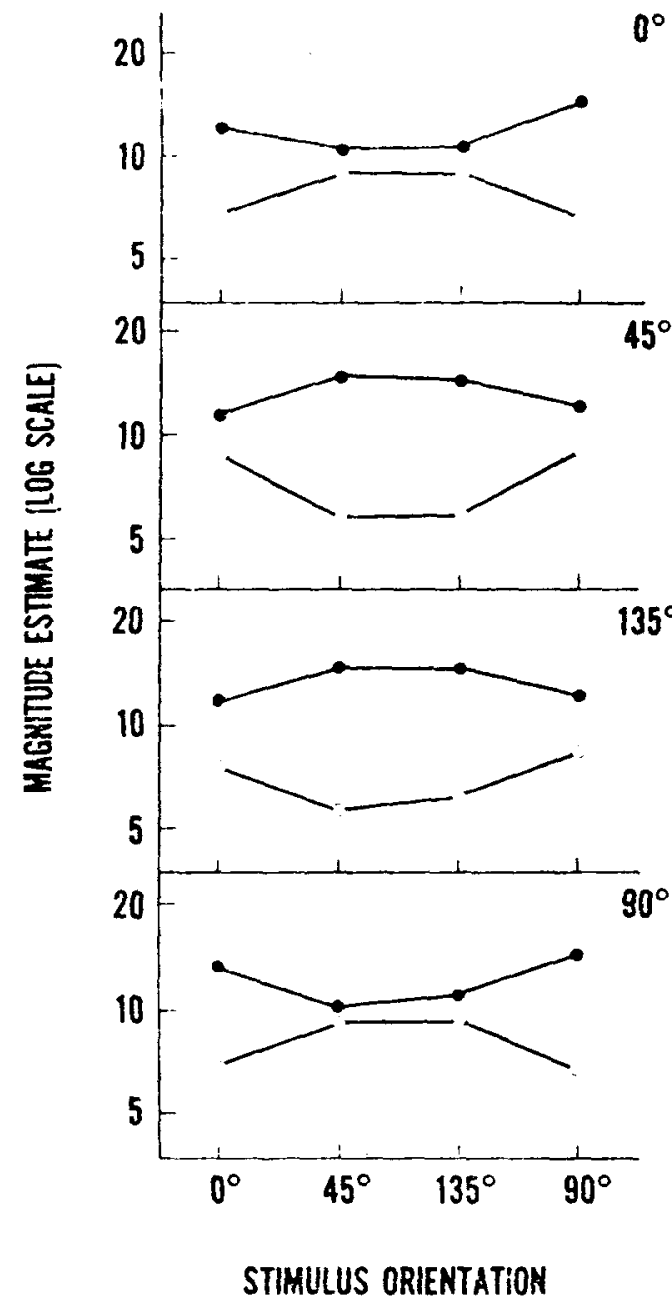

Fig. 2. Results of Experiment II. Magnitude estimates of the lightness of gray bars on black (filled circles) and white (open circles) backgrounds as a function of configuration orientation for four different positions of the head. Head orientations are given in the upper right of each graph.

The difference between means was reversed when the head was tilted obliquely. Against a white background, the magnitude of contrast was significantly greater for the oblique stimulus orientations (6.28) than for the horizontal-vertical orientations $(8.19)[F(1,48)=6.42$, $\mathrm{p}<.025]$.

Similarly, for the white background, significantly stronger contrast effects were found for the oblique stimulus orientations (14.29) than for the horizontal-vertical stimulus orientations (12.29) $[F(1,48)=7.26, p<.025]$.

The results, in Fig. 2, obtained with the head in a vertical (90 deg) orientation were analyzed separately from the rest of the data in this figure in order to determine whether these results replicated the findings of Experiment I. The results of this analysis indicate a significant effect of background $[F(1,4)=744.19$, $\mathrm{p}<.001]$. Black backgrounds enhance the lightness of 
gray bars. and white backgrounds depress the lightness of gray bars. as found in Experiment I also.

Similarly, a significant interaction between stimulus orientation and background lightness $[F(3,12)=12.98$, $p<.001]$ indicates that the difference in judgment for the horizontally oriented gray bar and vertically oriented gray bar on white and black backgrounds was greater than the difference in judgment for the obliquely oriented gray bars on white and black backgrounds.

Scheffé tests indicate that the horizontal and vertical differences are significant $[F(1,12)=57.76, p<.025$, and $F(1.12)=63.57, p<.025$, respectively], while the differences of the oblique stimulus orientations, right oblique (45 deg), and left oblique (135 deg) are not significant $[F(1,12)=1.58, p>.025$, and $F(1,12)=$ $3.56, \mathrm{p}>.025$, respectively].

Consequently, the lightness judgments obtained with the head in a vertical position in Experiment II replicate the findings obtained in Experiment $I$ with the head in a similar orientation.

\section{DISCUSSION}

The effects of stimulus orientation on lightness contrast were studied in order to determine whether the visual mechanism responsible for the analysis of contrast was cortical. The results of this investigation indicate the following: (1) lightness contrast depends upon stimulus orientation, and (2) it is retinal orientation rather than environmental orientation which is important.

It was argued that orientation effects should not be present if contrast analysis is determined at a subcortical locus (i.e., retinal, LGN) in the visual system, since receptive fields at those levels are concentrically organized and will fire with the same "strength" to a bar in any orientation. Cortical cells, however, exhibit orientation tuning, that is, the maximum firing is to a stimulus in a particular orientation (Hubel \& Wiesel, 1968). More importantly, electrophysiological experiments have demonstrated that the firing rate of cells in the visual cortex are stronger for stimuli presented in horizontal and vertical orientations than for obliquely oriented stimuli (Pettigrew et al, 1968). As a consequence of this, one might expect that the effectiveness of backgrounds should be diminished, that the apparent difference between a gray bar on a black background and a gray bar on a white background should be less marked for stimuli presented in the oblique orientations than for horizontal and vertical orientation. Such a result can be seen in Fig. 1 .

It should be apparent from this discussion that the results obtained in this experiment are rather similar to those obtained in threshold tasks involving acuity or contrast sensitivity (Higgins \& Stultz, 1948; Leibowitz, 1953; Campbell \& Kulikowski, 1966; Gilinsky, 1968). In these experiments, the amount of light was determined in order to perceive a grating. In the oblique orientation, more light is needed or acuity was better in the horizontal and vertical meridians at the same luminance level. This is because the difference between the peaks and troughs in the grating is much less in the oblique meridians than in the horizontal and vertical ones. In the present study, the white and black backgrounds represent a peak and trough, respectively. Thus, in this experiment, we asked for judgments of the apparent differences of lightness between gray bars on a peak and in a trough for different stimulus orientations.

The apparent difference in performance for obliquely oriented stimuli has been found in a wide variety of perceptual tasks (see Appelle, 1972), and it seems that the locus of this "oblique effect" (Appelle, 1972) is cortical (Maffei \& Campbell, 1970; Campbell \& Kulikowski, 1966).

The implication of these results is that the analysis of contrast occurs at or after the locus in the visual cortex where stimulus orientation is analyzed. These results dovetail nicely with other studies of simultaneous contrast which indicate that retinal processing of contrast is probably incorrect (Hollins, 1971; Fiorentini \& Maffei, 1973) and are consistent with other psychophysical experiments that have suggested a cortical locus for contrast analysis (Yund, 1970; Gogel \& Mershon, 1969; Mershon, 1972; Wist \& Susen, in press).

\section{REFERENCES}

Alpern, M. Metacontrast. Journal of the Optical Society of America, 1953, 43, 648-657.

Appelle, S. Perception and discrimination as a function of stimulus orientation. Psychological Bulletin, 1972, 78, 266-278.

Barlow, H. B., Fitzhugh, R., \& Kuffler, S. W. Change of organization in the receptive fields of the cat's retina during dark adaptation. Journal of Physiology, 1957, 137. 338-354.

Blakemore, $C_{\text {, }}$ \& Campbell, $F$. W. On the existence of neurones in the human visual system selectively sensitive to the orientation and size of retinal images. Journal of Physiology, $1969,203,237-260$.

Campbell, F. W., \& Maffei, L. Electrophysiological evidence for the existence of orientation and size detectors in the human visual system. Journal of Physiology, 1970, 207, 635-652.

Campbell, F. W., \& Kulikowski, J. J. Orientation selectivity of the human visual system. Journal of Physiology, 1966, 187, $437-445$

Fiorentini, A., \& Maffei, L. Contrast in night vision. Vision Research, 1973, 13, 73-80.

Gilinsky, A. S. Orientation-specific effects of patterns of adapting light on visual acuity. Journal of the Optical Society of America, 1968, 58, 13-18.

Gogel, W. C., \& Mershon, D. H. Depth adjacency in simultaneous contrast. Perception \& Psychophysics, 1969, 5, 13-17.

Higgins, G. C., \& Stultz, K. Visual acuity as measured with various orientations of a parallel line test object. Journal of the Optical Society of America, 1948, 38, 756-758.

Hollins, M. Brightness contrast at low luminances. Vision Research, 1971, 11, 1459-1472.

Hubel, D. H., \& Wiesel, T. N. Receptive fields, binocular interaction and functional architecture in the cat's visual cortex. Journal of Physiology, 1962, 160, 106-154. 
Hubel. D. H.. \& Wiesel, T. N. Receptive fields and functional architecture of monkey striate cortex. Journal of Physiology. $1968,195.215-243$.

Leibowitz. H. Some observations and theory on the variation of visual acuity with the orientation of the test object. Journal of the Optical Society of America, 1953, 43, 902-905.

Maffei, L., \& Campbell, F. W. Neurophysiological localization of the vertical and horizontal visual coordinates in man. Science, 1970, 167, 386-387.

Mershon. D. H. Relative contributions of depth and directional adjacency to simultaneous whiteness contrast. Vision Research. 1972. 12, 969-979.
Pettigrew, J. D.. Nikara, T. \& Bishop. P. O. Responses to moving slits by single units in cat striate cortex. Experimental Brain Research, 1968, 6, 373-390.

Wist, E. R., \& Susen. P. Evidence for the role of post-retinal processes in simuitaneous contrast. Psychologische Forschung. in press.

Yund, W. Edge-distance model for simultaneous contrast. PhD thesis, Northeastern University, 1970.

(Received for publication April 16, 1973; revision received June 18,1973 .) 